\title{
Blood Pressure Lowering Decreases Intracerebral Hemorrhage Volume and Improves Behavioral Outcomes in Experimental Animals
}

\author{
Ferdinand O. Bohmann, ${ }^{\text {a,b }}$ Alexander Seiler, ${ }^{\text {a }}$ Sarah Gelhard, ${ }^{\mathrm{a}}$ Leonie Stolz, ${ }^{\mathrm{a}}$ Boris Brill, ${ }^{\mathrm{c}}$ \\ Robert Brunkhorst, ${ }^{\mathrm{a}}$ Helmuth Steinmetz, ${ }^{\mathrm{a}}$ Patrick N. Harter, ${ }^{\mathrm{b}}$ Michel Mittelbronn, ${ }^{\mathrm{b}, \mathrm{d}, \mathrm{e}, \mathrm{f}, \mathrm{g}}$ \\ Christian Foerch ${ }^{\mathrm{a}}$ \\ aDepartment of Neurology, Goethe University, Frankfurt am Main, Germany \\ 'Institute of Neurology (Edinger-Institute), Goethe University, Frankfurt am Main, Germany \\ 'Georg-Speyer-Haus, Institute for Tumor Biology and Experimental Therapy, Frankfurt am Main, Germany \\ 'Luxembourg Centre of Neuropathology (LCNP), Luxembourg City, Luxembourg

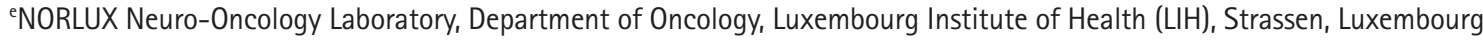 \\ fLuxembourg Centre for Systems Biomedicine (LCSB), University of Luxembourg, Esch-sur-Alzette, Luxembourg \\ ${ }^{9}$ Department of Pathology, Laboratoire National de Santé (LNS), Dudelange, Luxembourg
}

\section{Dear Sir:}

Arterial blood pressure is often elevated in the acute phase of intracerebral hemorrhage (ICH). It is a predictor of a poor prognosis. ${ }^{1}$ The Intensive Blood Pressure Reduction in Acute Cerebral Hemorrhage Trial II (INTERACT II) study revealed a strong tendency towards improved outcomes in $\mathrm{ICH}$ patients subjected to intensive blood pressure lowering, ${ }^{1}$ while the Antihypertensive Treatment of Acute Cerebral Hemorrhage Trial II (ATACH II) trial reported worse outcomes. ${ }^{2}$ So far, the pathophysiologic consequences of intensive blood pressure lowering in acute ICH are scarcely understood. Here we describe an experimental model with translational reference to the INTERACT II and ATACH II trials. Our study allows for rapid blood pressure lowering 30 minutes after $\mathrm{ICH}$ induction in rats. We aimed at assessing hematoma volume and functional outcome and evaluated histological and magnetic resonance imaging (MRI) parameters, including edema formation and blood-brain barrier dysfunction.

For this study 84 spontaneously hypertensive rats (SHR) were used. A detailed methodical description can be found in Supplementary methods. In brief, striatal ICH was induced by stereotactic collagenase injection. ${ }^{3,4}$ SHR were then randomized into an interventional group (intensive blood pressure lowering by nifedipine orally to $126.0 \pm 8.8 \mathrm{~mm} \mathrm{Hg}$ systolic) and a control group (saline orally; $186.0 \pm 9.8 \mathrm{~mm}$ Hg systolic) (Supplementary Figure 1). Hematoma volume as the primary endpoint was quantified photometrically 24 hours after $\mathrm{ICH}$ induction (end of main hematoma expansion phase). Neurological deficits were evaluated before sacrifice by a blinded rater using the modified Neurological Severity Score (mNSS). Additional histopathological analysis evaluated edema formation and blood brain barrier dysfunction at 24 and 72 hours after ICH induction (Supplementary Table 1). Ordinal histoscores were calculated using a semi-quantitative assessment (Supplementary methods 1).

Intensive blood pressure lowering reduced hematoma volume 24 hours after ICH induction (nifedipine group 88.6 \pm 36.6 $\mu \mathrm{L}$ vs. control group $146.3 \pm 58.7 \mu \mathrm{L}, P=0.023, \mathrm{n}=9$ ) (Figure $1 \mathrm{~A}$ ). This implicated a decrease in hematoma growth within the first 24 hours by nifedipine. Neurological deficits were significantly reduced 24 hours after ICH induction in the interventional group as compared to controls (median, 7 [interquartile range, IQR, 5.5 to 11$]$ vs. 13 [IQR, 9.5 to 18$] ; P=0.011, n=9$ ) (Figure 1B).

Histopathological analyses revealed no differences in edema formation at 24 hours (histoscore: nifedipine median, 2 [IOR, 2 to 3 ] vs. control 3 [IOR, 2 to 3$] ; P=0.99, \mathrm{n}=5$ ) and 72 hours 

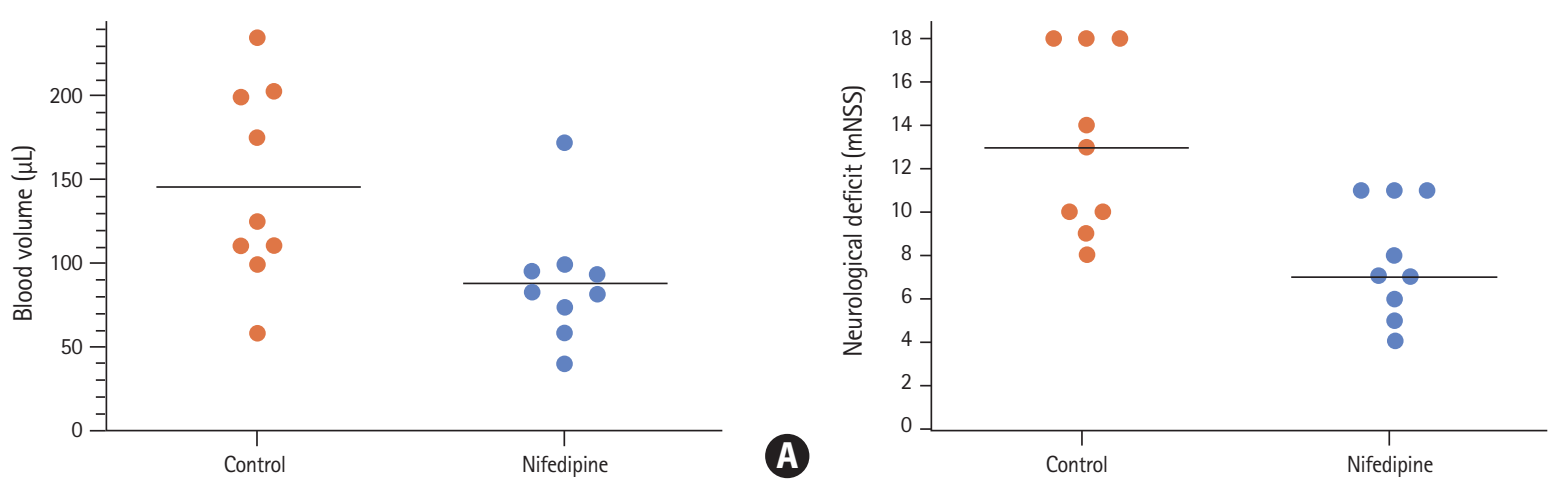

B

Figure 1. (A) Hematoma volume $(\mu \mathrm{L}) 24$ hours after intracerebral hemorrhage $(\mathrm{ICH})$ induction in rats subjected to intensive blood pressure lowering (nifedipine) versus controls $(P=0.02, n=9)$. Individual data points and mean values are given. (B) Neurological deficits 24 hours after ICH induction in rats subjected to intensive blood pressure lowering (nifedipine) versus controls $(P=0.01)$. Individual data points and median values are given. mNSS, modified Neurological Severity Score.

(nifedipine median, 1 [IQR, 0.5 to 2] vs. control 1 [IQR, 0.5 to 2], $P=0.99, n=5$ ) (Supplementary Figures 2 and 3). Concerning the extent of blood-brain barrier disruption the size of the areas showing positive immunoglobulin $\mathrm{G}$ staining did not differ between groups at 24 hours (histoscore: nifedipine median, 3 [IOR, 3 to 4] vs. control 3 [IOR 2 to 4$], P=0.99, n=5)$ and 72 hours (nifedipine, 3 [IQR, 2 to 3] vs. control 2 [IQR, 1.5 to 3], $P=0.68, n=5$ ) (Supplementary Figures 2 and 3). An additional descriptive MRI of post-mortem rat brains confirmed findings (Supplementary Figure 4).

Our experimental data suggest that intensive blood pressure lowering in the acute phase of $\mathrm{ICH}$ reduces hematoma volume and improves functional neurological deficits. We did not find a significant effect of the treatment on edema formation and blood-brain barrier dysfunction up to 72 hours after ICH induction.

From a translational perspective, the rapid drop of blood pressure after nifedipine administration was well comparable to what has been achieved in clinical trials (although different in its absolute size). ${ }^{1,2}$ We consider it unlikely that nifedipine has other effects on hematoma volume and functional outcome than what is mediated via blood pressure. Moreover, calcium channel blockers were also used in the pivotal clinical trials. ${ }^{1,2}$ In experimental ICH, bleeding begins around 10 minutes after collagenase injection. Thus, our model well covers blood pressure lowering during the phase of hematoma expansion. ${ }^{3-5}$

The effect of rapid blood pressure lowering on functional outcome after ICH is not consistent between clinical and translational studies. On the clinical side, INTERACT II reported a strong trend towards a benefit of the intervention whereas ATACH II reported neutral or even worse outcomes. ${ }^{1,2}$ The ani- mal data in contrast affirms that blood pressure reduction translates into a better functional outcome. In fact, in the ATACH II trial more complications occurred in the intensively treated group such as increased frequency of kidney failure. ${ }^{1,2}$ However, our model was not designed to capture systemic complications of intensive blood pressure lowering.

We did not observe differences in other pathophysiological cascades that take place in the perilesional tissue after $\mathrm{ICH}$ formation (especially edema formation and blood brain barrier disruption). It appears that the most critical mediator of the therapeutic effect of intensive blood pressure lowering is the reduction in hematoma volume. However, our model can be applied to future studies evaluating the pathophysiology of blood pressure lowering in acute ICH in more detail.

Limitations include missing longitudinal outcome data to prove long-term therapeutic effects (in line with 90 days outcome of the randomized trials) of the intervention. Furthermore, we may have overestimated the therapeutic effect size of the intervention because the control group was untreated, thus having higher blood pressure values than the control groups of the pivotal clinical trials. ${ }^{1,2}$

In conclusion, blood pressure lowering with nifedipine decreases ICH volume and improves behavioral outcomes in SHR. Our findings may help to refine future clinical studies on blood pressure reduction in ICH patients.

\section{Supplementary materials}

Supplementary materials related to this article can be found online at https://doi.org/10.5853/jos.2020.02390. 


\section{References}

1. Anderson CS, Heeley E, Huang Y, Wang J, Stapf C, Delcourt C, et al. Rapid blood-pressure lowering in patients with acute intracerebral hemorrhage. N Engl J Med 2013;368:23552365.

2. Qureshi Al, Palesch YY, Barsan WG, Hanley DF, Hsu CY, Martin $\mathrm{RL}$, et al. Intensive blood-pressure lowering in patients with acute cerebral hemorrhage. N Engl J Med 2016;375:10331043.

3. Rosenberg GA, Mun-Bryce S, Wesley M, Kornfeld M. Collagenase-induced intracerebral hemorrhage in rats. Stroke 1990; 21:801-807.

4. Foerch C, Arai K, Jin G, Park KP, Pallast S, van Leyen K, et al. Experimental model of warfarin-associated intracerebral hemorrhage. Stroke 2008;39:3397-3404.

5. hou W, Schwarting S, Illanes S, Liesz A, Middelhoff M, Zorn
$M_{1}$ et al. Hemostatic therapy in experimental intracerebral hemorrhage associated with the direct thrombin inhibitor dabigatran. Stroke 2011;42:3594-3599.

Correspondence: Ferdinand 0. Bohmann

Department of Neurology, Goethe University, Schleusenweg 2-16, 60528 Frankfurt am Main, Germany

Tel: +49-69-6301-4406

Fax: +49-69-6301-7084

E-mail: ferdinand.bohmann@kgu.de https://orcid.org/0000-0001-6439-0667

Received: June 17, 2020

Revised: August 19, 2020

Accepted: August 21, 2020

Michel Mittelbronn would like to thank the Luxembourg National Research Fond (FNR) for the support (FNR PEARL P16/BM/11192868 grant). This study was supported in part by a grant from the Goethe University Hospital Frankfurt.

The authors have no financial conflicts of interest. 
Supplementary Table 1. Study design and animals per experimental group

Photometric hematoma quantification and 9 SHR per group ( $n=18)$ (primary endpoint)

functional outcome (mNSS)

\begin{tabular}{lll}
$\begin{array}{l}\text { Histopathological analysis } \\
\text { - HE staining }\end{array}$ & 5 SHR per group $(n=10)$ & 5 SHR per group $(n=10)$ \\
- Immunohistochemical analysis & \\
Explorative post-mortem MRI acquisition & 3 SHR per group $(n=6)$ & 3 SHR per group $(n=6)$ \\
\hline
\end{tabular}

Blood pressure evaluation was done separately in 8 SHR in total.

ICH, intracerebral hemorrhage; mNSS, modified Neurological Severity Score; SHR, spontaneously hypertensive rats; HE, hematoxylin eosin; MRI, magnetic resonance imaging. 

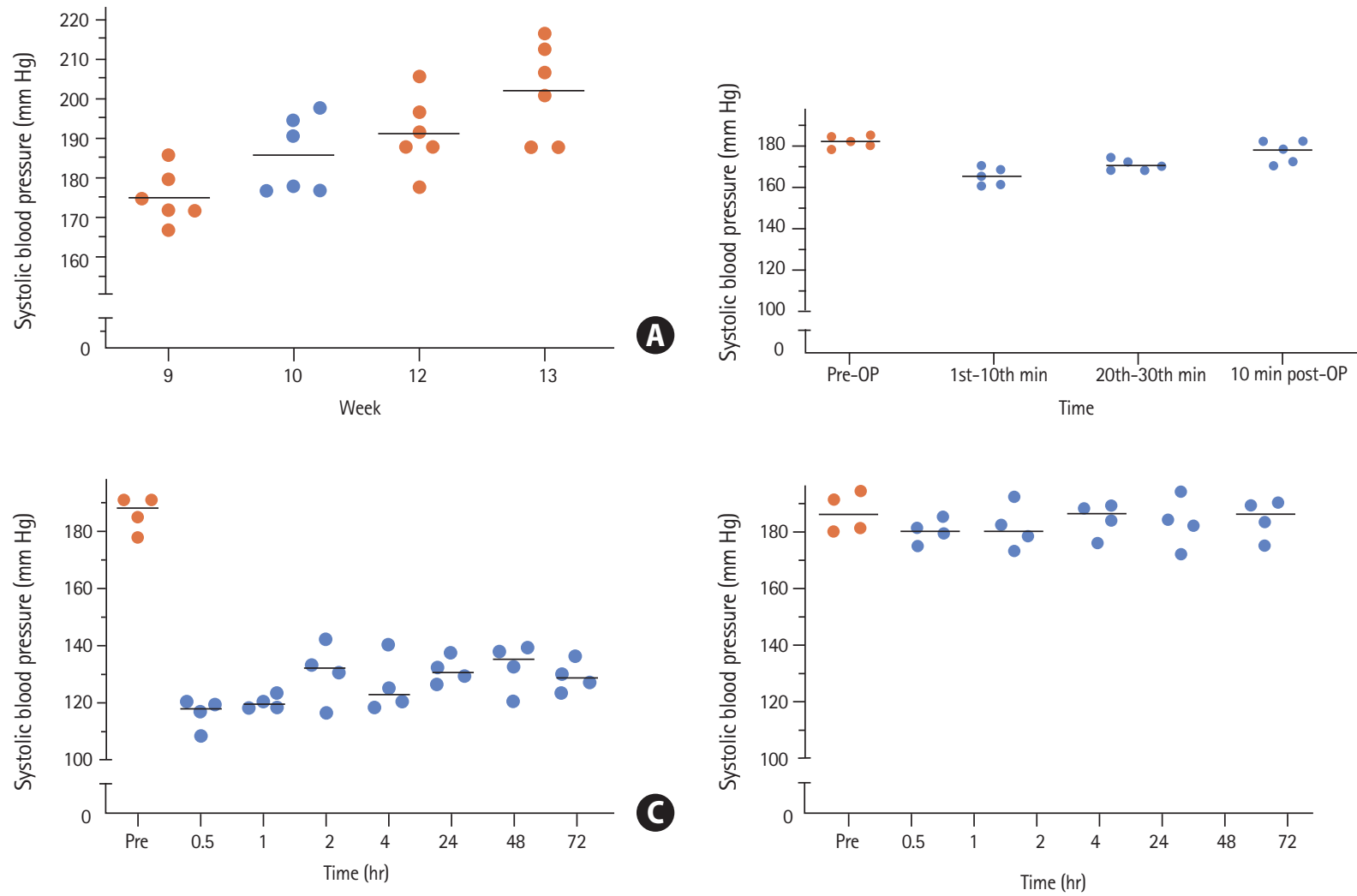

Supplementary Figure 1. Blood pressure evaluation (tail-cuff method). (A) Systolic blood pressure values (mm Hg) of spontaneously hypertensive rats stratified according to age (weeks). In addition to individual data points, mean values are given (black lines). Intracerebral hemorrhage experiments were performed with rats aged 10 weeks (blue symbols). (B) Perioperative systolic blood pressure values. Systolic blood pressure values (mm Hg) before (pre-operative, red symbols), during (1st-10th minute, 20th-30th minute, blue symbols) and after (post-operative, blue symbols) isoflurane anesthesia. In addition to individual data points, mean values are given (black lines). (C) Blood pressure lowering with nifedipine orally over 72 hours (intervention). Systolic blood pressure values $(\mathrm{mm} \mathrm{Hg})$ before (red symbols) and during the application of nifedipine $(15 \mathrm{mg} / \mathrm{kg}$ body weight, blue symbols) via a nasogastric tube (every 8 hours during the first 24 hours, every 12 hours thereafter). In addition to individual data points, mean values are given (black lines). (D) Blood pressure levels during placebo application orally over 72 hours (control group). Systolic blood pressure values $(\mathrm{mm} \mathrm{Hg})$ before (red symbols) and during the application of saline as placebo via a nasogastric tube (every 8 hours during the first 24 hours, every 12 hours thereafter). Treatment and application volume were equal to nifedipine-treated rats. In addition to individual data points, mean values are given (black lines).

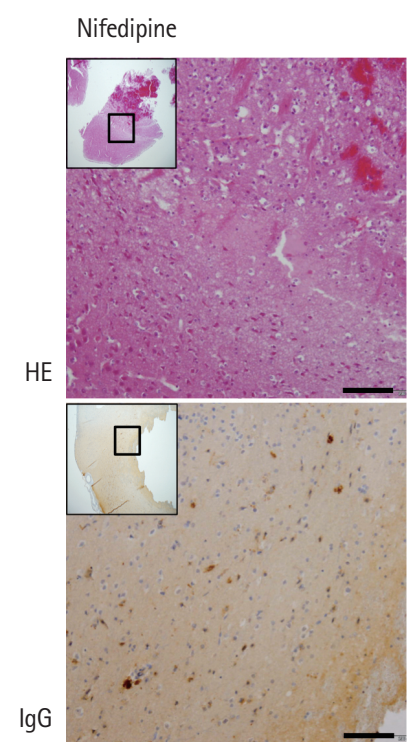

Control

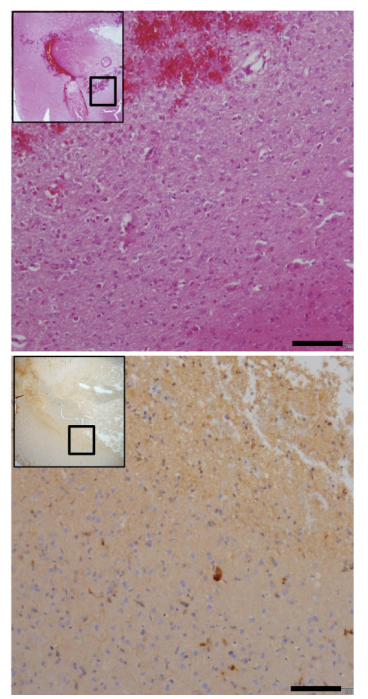

Supplementary Figure 2. Representative hematoxylin eosin (HE) and antirat-immunoglobulin $\mathrm{G}(\mathrm{lgG})$ staining sections for the intensive blood pressure lowering vs. control group. Representative HE and anti-rat-IgG staining sections for the intensive blood pressure lowering group (nifedipine, left side) and the control group (right side). No differences were found for edema formation (determined on HE sections) and for blood brain barrier dysfunction (as determined by anti-rat lgG immunohistochemical stainings) between the groups. Whole brain sections are depicted at original magnification $10($ bars $=200 \mu \mathrm{m})$ and smaller images with magnification 2 in the upper left corner for orientation. 

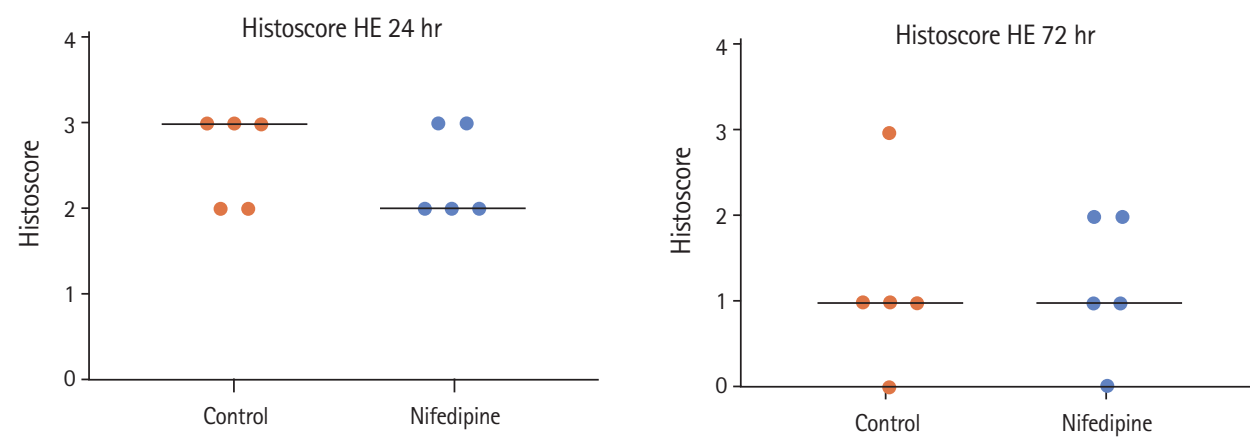

A
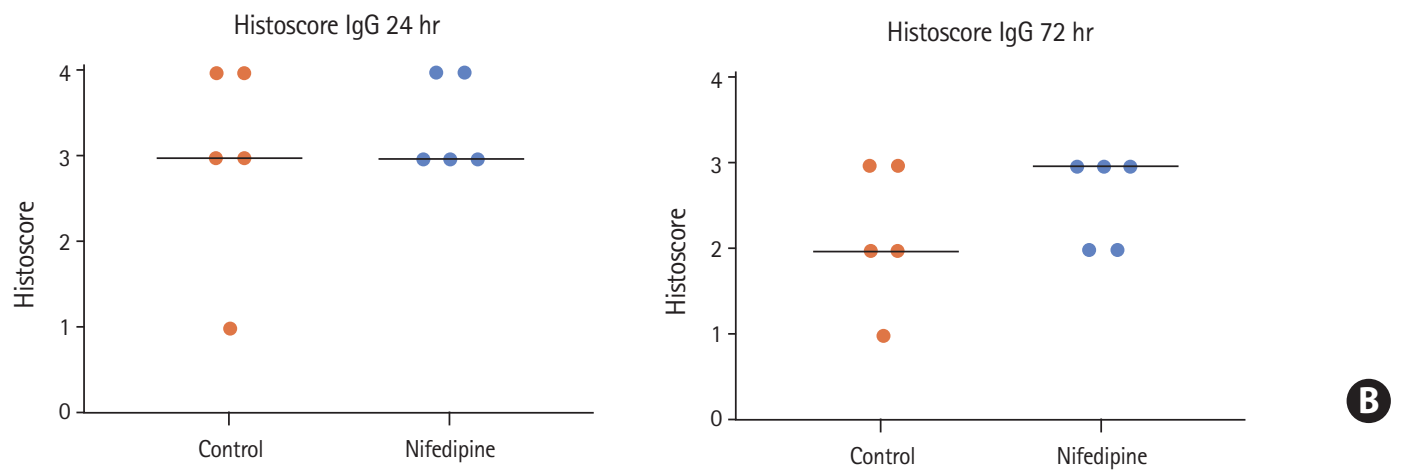

Supplementary Figure 3. Histopathological analysis using a semiquantitative histoscore. Ordinal histoscores were calculated using a semi-quantit ative assessment for edema formation (hematoxylin eosin [HE] staining, brain edema reflected by vacuolated tissue around the hemorrhage compatible with vasogenic brain edema: $0=$ isolated hemorrhage without vacuolated tissue, reflecting brain edema; $1=$ edema minimal, means partial vacuolated tissue around

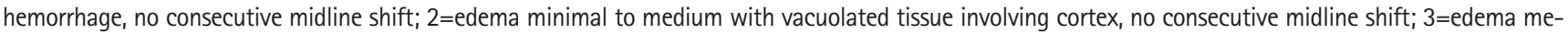
dium to strong with consecutive midline shift; $4=$ strong edema bilateral, midline shift) and blood brain barrier dysfunction (immunoglobulin $\mathrm{G}$ [lgG] staining, intraparenchymatous deposits of $\lg \mathrm{G}$ as indirect marker of blood brain barrier dysfunction: $0=$ =isolated hemorrhage, extravasation absent; $1=$ extravasation and

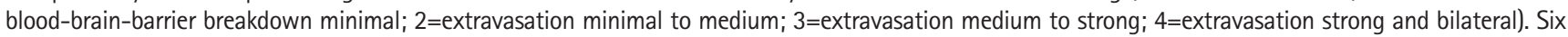
slides per brain prepared from needle entry site $\pm 1 \mathrm{~mm}$ were analyzed. The slides were evaluated in blinded random order with a standard light microscopy. (A) No differences in edema formation at 24 hours $(P=0.99, n=5)$ and 72 hours $(P=0.99, n=5)$. (B) Concerning the extent of blood-brain barrier disruption the size of the areas showing positive lgG staining did not differ between groups at 24 hours $(P=0.99, n=5)$ and 72 hours $(P=0.68, n=5)$. Median scores and scatter dot plot are presented.
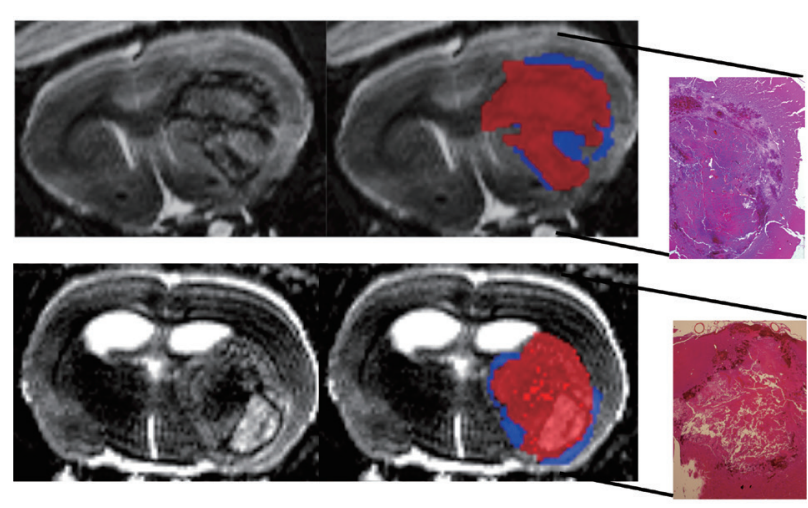

A

B
Supplementary Figure 4. Explorative magnetic resonance imaging (MRI)based edema quantification 24 hours after intracerebral hemorrhage (ICH) induction. Representative magnetic resonance image (T2) of two fixed rat heads 24 hours after ICH induction with comparable hematoma volume around $90 \mu \mathrm{L}$. (A) Control group without blood pressure lowering and (B) with intensive blood pressure lowering (nifedipine-treated). The calculated edema volume in both MRIs was around $20 \mu \mathrm{L}$ (blue area). Afterwards histopathological analysis (hematoxylin eosin [HE] staining) from the same brains confirmed imaging results. 


\section{Supplementary methods}

\section{Animals}

For this study 84 spontaneously hypertensive rats (SHRs) were used (10 weeks old, male, body weight approximately $260 \mathrm{~g}$; Charles River Laboratories, Sulzbach, Germany). SHR constitute a well-established hypertensive rat model. ${ }^{1,2}$ All experimental procedures were conducted in accordance with the National Institutes of Health's guide for the care and use of laboratory animals. The report of the data in this manuscript is compliant with the Animal Research: Reporting of In Vivo Experiments (ARRIVE) guidelines. ${ }^{3}$ This study was approved from the responsible regulatory authorities in Germany (Regierungspraesidium Darmstadt, approval number FU/1070).

\section{Study design and sample size calculation}

Previous studies showed a significantly worse functional outcome for hypertensive vs. normotensive rats when intracerebral hemorrhage $(\mathrm{ICH})$ was induced. ${ }^{4}$ Thus, we assumed a beneficial effect of an intensive blood pressure lowering strategy after $\mathrm{ICH}$. Rats were randomized into an interventional (intensive blood pressure lowering) group and a control group, respectively. Besides anti-hypertensive treatment, both groups were treated identically. Hematoma volume and neurological deficits were determined 24 hours after $\mathrm{ICH}$ induction (time point of expected maximum hemorrhage expansion and neurological impairment). Histopathological analysis and magnetic resonance imaging (MRI) were performed 24 and 72 hours after $\mathrm{ICH}$ induction (to enhance sensitivity for secondary injury after ICH during the first 3 days). An overview of animal numbers per time point can be found in the Supplementary Table 1.

Based on the effect size of our previous studies (Cohens $D=1.75),{ }^{5}$ we needed at least seven rats per group in order to detect a significant difference in hematoma volume between the treatment regimens with a power of $80 \%$ and a type 1 error of 0.05 using a two-tailed Student's t-test. Thus, we randomized nine rats per group.

\section{ICH induction}

All surgery was performed under isoflurane anesthesia (1.5\% to $2 \%$ ) with spontaneous respiration. For analgesia, $0.1 \mathrm{mg} / \mathrm{kg}$ buprenorphine were applied. For ICH induction, a small borehole was drilled and a 30-gauge $5 \mu \mathrm{L}$-microinjection needle (Hamilton, Bonaduz, Switzerland; 75RN series) was slowly lowered into the right striatum at the following coordinates from bregma: $0.2 \mathrm{~mm}$ anterior, $3.5 \mathrm{~mm}$ lateral, and $5.5 \mathrm{~mm}$ depth. A $2 \mu \mathrm{L}$ of saline containing $0.25 \mathrm{U}$ collagenase VII-S (Sigma,
Darmstadt, Germany) or saline alone were injected over 5 minutes using a microinfusion pump (Quintessential Stereotaxic Injector, Stoelting Co., Wood Dale, IL, USA). The needle was left in place for 10 minutes and was then slowly removed. Afterwards, the borehole was sealed with bone wax, the scalp was closed, and the rats were allowed to recover. The duration of the whole surgical procedure was approximately 35 minutes for each rat. A closed-loop temperature system with a heating pad and a rectal temperature probe was used to maintain body temperature.

\section{Blood pressure lowering in acute $\mathrm{ICH}$}

Intensive blood pressure management in acute $\mathrm{ICH}$ requires specific qualities for the drug of choice: (1) orally applicable to avoid stress induced hypertension; (2) quickly effective to ensure rapid blood pressure lowering after ICH induction; (3) non-sedating to allow neurological monitoring. Unlike clonidine, nifedipine fulfils these criteria without relevant side effects. Nifedipine is a dihydropyridine calcium channel blocker. The anti-hypertensive effects of nifedipine in rats administered by chow for up to 20 weeks are well characterized, no interaction with collagenase VII-S is known. ${ }^{6}$

The intensive blood pressure lowering group received nifedipine (oral liquid, $4 \mathrm{mg} / \mathrm{mL}$, Ratiopharm, Ulm, Germany) per nasogastric tube. Based on the results of explorative tests, a dosage of $15 \mathrm{mg} / \mathrm{kg}$ body weight with an application volume of $0.2 \mathrm{~mL}$ per treatment was chosen. In the control group saline alone was administered in the same frequency and volume. The first oral administration of nifedipine or saline, respectively, was scheduled at 30 minutes after $\mathrm{ICH}$ induction, to ensure the swallowing reflex. Thereafter, rats were fed every 8 hours during the first 24 hours, then every 12 hours.

Arterial blood pressure was measured non-invasively using a photoelectric tail cuff device (BP 2000, blood pressure system for rats, Biomedical Instruments, Zöllnitz, Germany). This method has previously been validated in SHR. ${ }^{2,7}$ Rats were placed in a restrainer on a heating plate with a temperature of $36^{\circ} \mathrm{C}$. A cuff was attached to the tail and gently tightened until detection of the pulse signal. The animals acclimatized on the plate for 10 minutes, followed by 10 pre-measurements to reduce stress level. Ten measurements were then performed to generate mean values for systolic and diastolic blood pressure. To avoid any impact of tail cuff measurements during critical postoperative hours, blood pressure was not evaluated in animals scheduled for hematoma quantification, histopathological analysis or outcome assessment. Instead, we analyzed separate animals after nifedipine/saline treatment up to 72 hours to en- 
sure stable blood pressure lowering in the intervention group.

\section{Functional outcome}

Neurological deficits were evaluated by a blinded rater 24 hours after ICH induction using the modified Neurological Severity Score (mNSS). ${ }^{8,9}$ The 18-point mNSS includes testing for hemiparesis, gait, coordination and sensory functions. Videotaped sequences were assessed for spontaneous motion activity and the beam walking test, both parts of the mNSS. Rats were not trained before the tests. Rats that died within the observation period were assigned the maximum of 18 points.

\section{Determination of ICH volume}

After mNSS assessment, rats were transcardially perfused with $50 \mathrm{~mL}$ phosphate buffered saline (PBS) under deep isoflurane anesthesia. Hemoglobin concentration was measured for each hemisphere separately following a previously described protocol. ${ }^{5}$ In brief, hemispheres were homogenized, subjected to ultrasound for 60 seconds and centrifuged $\left(13.000 \mathrm{rpm}, 4^{\circ} \mathrm{C}, 30\right.$ minutes). Photometric analysis of the supernatant mixed with Drabkin's Reagent solution (Sigma-Aldrich, Taufkirchen, Germany) was then performed in duplicates at $540 \mathrm{~nm}$. ICH volume was calculated based on a standard curve (data not shown in detail).

Rats found dead within the observation period could not undergo transcardial perfusion. In this case, we performed a total autopsy to exclude extracerebral bleeding. Afterwards, we determined ICH volume as described above and subtracted 2.4 $\mu \mathrm{L}$. This value was found to be the estimated cerebral blood volume in $\mathrm{SHR}^{10}$

\section{Histopathological analysis}

After transcardial perfusion with $40 \mathrm{~mL}$ PBS and $40 \mathrm{~mL}$ buffered formalin ( $\mathrm{pH}$ 7.4), rat brains were stored in buffered formalin and embedded in paraffin. Fixed brains were cut through the needle entry site (identifiable on the brain surface) as well as stepwise every $1 \mathrm{~mm}$ anterior and posterior to that plane. Sections $(3 \mu \mathrm{m})$ were obtained using a microtome (Quintessential Stereotaxic Injector, Stoelting), placed on SuperFrost Plus slides, and deparaffinized. Hematoxylin eosin (HE) staining was performed. After incubation, slides were mounted in isopropanol followed by xylene.

Immunhistochemical analysis was performed on the Discovery XT Immunohistochemistry System (Ventana, Strasbourg, France) as previously described. ${ }^{11}$ The following anti-rat antibodies were applied: rabbit anti-immunoglobulin $\mathrm{G}(\mathrm{lgG})(\mathrm{H} \& \mathrm{~L}$, ab6703, dilution 1:2,000, Abcam, Cambridge, UK), and mouse anti-major histocompatibility complex class II antibody (MRC
OX-6, ab23990, dilution 1:500, Abcam). Sections were washed, counterstained with hematoxylin and bluing reagent and mounted. Evaluation of the stainings and photographic documentation were performed using an Olympus BX-50 light microscope (Hamburg, Germany).

Ordinal histoscores were calculated using a semi-quantitative assessment for edema formation (HE staining, vacuolated tissue compatible with vasogenic brain edema: $0=$ isolated hemorrhage, edema absent; $1=$ edema minimal, no midline shift; $2=$ =edema minimal to medium with cortical involvement, no midline shift; $3=$ =edema medium to strong with midline shift; $4=$ strong edema bilateral, midline shift) and blood brain barrier dysfunction ( $\lg G$ staining, $\lg G$ extravasation as indirect marker of blood brain barrier dysfunction: $0=$ =isolated hemorrhage, extravasation absent; $1=$ extravasation and blood-brain-barrier breakdown minimal; $2=$ extravasation minimal to medium; $3=$ extravasation medium to strong; $4=$ extravasation strong and bilateral). Six slides per brain prepared from needle entry site $\pm 1 \mathrm{~mm}$ were analyzed. The slides were evaluated in blinded random order with a standard light microscopy. Median scores were calculated. ${ }^{12}$

\section{Post-mortem MRI}

Post-mortem MRI was applied as described elsewhere. ${ }^{13}$ Following an identical experimental procedure as described above, $40 \mathrm{~mL}$ warmed saline-Prohance (in $0.9 \%$ saline, $37^{\circ} \mathrm{C}, 1: 10$ solution of Prohance, Bracco, Princeton, NJ, USA) was used for perfusion (4 minutes). Then $40 \mathrm{~mL}$ formalin-Prohance (in 10\% formalin, 1:10 solution of Prohance, Milano, Italy) was given for another 4 minutes. Whole rat heads were removed and put into 10\% formalin for 24 hours without Prohance. Heads were then transferred into Prohance-PBS (1:100 solution) and stored at $4^{\circ} \mathrm{C}$. MRI was performed using a 7 Tesla small animal scanner (PharmaScan, Bruker, Ettlingen, Germany) with a volume coil. Data acquisition was performed using the Paravision 6.0.1 software (Bruker, Billerica, MA, USA). Modified driven equilibrium Fourier transform (MDEFT) sequence was used for T1-weighted images with the following parameters: echo time $(T E)=2 \mathrm{~ms}$, relaxation time $(T R)=4,000 \mathrm{~ms}$. The matrix size was $256 \times 256$ and the voxel size $0.17 \mathrm{~mm}$. T2 sequences were measured afterwards ( $T R=2,200 \mathrm{~ms} ; \mathrm{TE}=33 \mathrm{~ms}$ ). The matrix size was $512 \times 512$ and the voxel size $0.08 \mathrm{~mm}$. Total imaging time was 3 hours per rat head. Data analysis was performed by a blinded rater slide by slide quantifying hematoma and edema volume on T1 and T2-weighted images (MRIcro, Chris Rorden, Columbia, SC, USA; www.mricro.com). 


\section{Statistical analysis}

Prism 7 (GraphPad Software, San Diego, CA, USA) and SPSS version 22.0 (IBM Co., Armonk, NY, USA) were used for statistical analysis. Blood pressure levels are presented as mean \pm standard deviation (unless otherwise stated, systolic blood pressure values are mentioned). Hematoma volume results are displayed in a scatter dot plot with mean values. Statistical significance was assessed using the two-tailed t-test after passing Shapiro-Wilk test. Neuroscore data are displayed in scatter plots with median values and interquartile range (IQR). Statistical significance was assessed using the Mann-Whitney U-test. For histoscore data medians and IQR are given and statistical significance was assessed using the Mann-Whitney U-test. Statistical significance was set to $P<0.05$.

\section{Supplementary References}

1. Palmer BM, Chen Z, Lachapelle RR, Hendley ED, LeWinter MM. Cardiomyocyte function associated with hyperactivity and/or hypertension in genetic models of LV hypertrophy. Am J Physiol Heart Circ Physiol 2006;290:H463-H473.

2. Pfeilschifter $W$, Steinstraesser T, Paulus P, Zeiner PS, Bohmann $F_{\text {, Theisen }} A$, et al. Risk of long-term anticoagulation under sustained severe arterial hypertension: a translational study comparing warfarin and the new oral anticoag ulant apixaban. J Cereb Blood Flow Metab 2017;37:855-865.

3. Kilkenny C, Browne W, Cuthill IC, Emerson M, Altman DG; NC3Rs Reporting Guidelines Working Group. Animal research: reporting in vivo experiments: the ARRIVE guidelines. BrJ Pharmacol 2010;160:1577-1579.

4. Bhatia PM, Chamberlain R, Luo X, Hartley EW, Divani AA. Elevated blood pressure causes larger hematoma in a rat model of intracerebral hemorrhage. Trans/ Stroke Res 2012;3:428434.
5. Foerch $C_{\text {, Arai }}$, Jin $G$, Park $K P$, Pallast $S$, van Leyen $K$, et al. Experimental model of warfarin-associated intracerebral hemorrhage. Stroke 2008;39:3397-3404.

6. Motz W, Ploeger M, Ringsgwandl G, Goeldel N, Garthoff B, Kazda $S$, et al. Influence of nifedipine on ventricular function and myocardial hypertrophy in spontaneously hypertensive rats. J Cardiovasc Pharmacol 1983;5:55-61.

7. Kubota Y, Umegaki K, Kagota S, Tanaka N, Nakamura K, Kunitomo $\mathrm{M}$, et al. Evaluation of blood pressure measured by tail-cuff methods (without heating) in spontaneously hypertensive rats. Biol Pharm Bull 2006;29:1756-1758.

8. Chen J, Sanberg PR, Li Y, Wang L, Lu M, Willing AE, et al. Intravenous administration of human umbilical cord blood reduces behavioral deficits after stroke in rats. Stroke 2001;32: 2682-2688.

9. Bohmann F, Mirceska A, Pfeilschifter J, Lindhoff-Last $E_{\text {, }}$ Steinmetz $H$, Foerch $C$, et al. No influence of dabigatran anticoagulation on hemorrhagic transformation in an experimental model of ischemic stroke. PLoS One 2012;7:e40804.

10. Bianchi M, Bellini $G$, Hessan $H$, Kim KE, Swartz $C$, Fernandes M. Body fluid volumes in the spontaneously hypertensive rat. Clin Sci (Lond) 1981;61:685-691.

11. Pfeilschifter $W$, Bohmann $F$, Baumgarten $P$, Mittelbronn $M$, Pfeilschifter J, Lindhoff-Last $E_{1}$ et al. Thrombolysis with recombinant tissue plasminogen activator under dabigatran anticoagulation in experimental stroke. Ann Neurol 2012; 71:624-633.

12. Gibson-Corley KN, Olivier AK, Meyerholz DK. Principles for valid histopathologic scoring in research. Vet Pathol 2013;50: 1007-1015.

13. Oguz I, Yaxley R, Budin F, Hoogstoel M, Lee J, Maltbie E, et al. Comparison of magnetic resonance imaging in live vs. post mortem rat brains. PLoS One 2013;8:e71027. 